\title{
Du savoir vers le savoir-faire : évolution de la conception de la REDD+ et contraintes à sa mise en œuvre en Afrique centrale
}

\author{
Moïse Tsayem Demaze ${ }^{1}$, Roger Ngoufo $^{2}$, Paul Tchawa ${ }^{2}$ \\ 1 Géographe, Université du Maine, CNRS, UMR 6590 ESO, 72085 Le Mans cedex 9, France \\ 2 Géographe, Université de Yaoundé I, BP 755 Yaoundé, Cameroun
}

\author{
Mots-clés : \\ changement \\ climatique ; REDD+ ; \\ analyse \\ institutionnelle ; \\ interactions \\ science-politique \\ publique \\ environnementale ; \\ Afrique centrale
}

\section{Keywords:}

climate change;

REDD +; institutional

analysis;

environmental public

policy and science

interactions;

Central Africa

\begin{abstract}
Résumé - Cet article examine les liens entre l'évolution de la conception de la REDD+ et les travaux scientifiques publiés à son sujet. Un cadre analytique, inspiré de l'étude des interactions entre sciences et politiques environnementales et de l'analyse institutionnelle discursive, est utilisé. Trois principales phases sont distinguées. La phase pré-RED, entre 1997 et 2004, la phase RED-REDD+, entre 2005 et 2009 , et la phase actuelle de préparation à la mise en œuvre dans les pays en développement. Un focus est ensuite effectué sur l'Afrique centrale, pour rendre compte de l'opérationnalisation de la REDD+. La lecture comparative des plans de préparation ne met pas en évidence un savoir-faire spécifique pour réduire ou éviter la déforestation dans les pays concernés. Multiforme, le mode opératoire révèle des incohérences entre développement économique et protection des forêts, ainsi que des difficultés pratiques soulignant la nécessité de réformes foncières et de politiques d'aménagement du territoire.
\end{abstract}

\begin{abstract}
From knowledge to know-how: evolution of REDD+ design and constraints to its implementation in central Africa. This paper describes the genesis of REDD + in order to analyze the relationship between the evolution of its design and the scientific literature published about it between 1997 and 2014. An analytical framework, involving the study of interactions between science and environmental public policy, and the discursive institutional analysis, is used. Three main phases are outlined. The Pre-RED phase, between 1997 and 2004, is that of the emergence of the concept of "compensated reduction" of emissions. Phase 2, between 2005 and 2009, is that of the effective development of REDD +, with a rich scientific production. The third phase, which began in 2010, focused on REDD+ readiness, with an abundant scientific production that has started to evaluate REDD+ procedures and pilots initiatives in developing countries. The paper then reports on the preparation for REDD+ in Central Africa countries, from a comparative reading of the readiness preparation proposals. Although this preparation is stepped, the necessary or specific know-how which can help avoid or reduce deforestation is not clearly stated. This highligts the diffuse nature of REDD+ even in its operational phase. The know-how is focused more on technical aspects related to monitoring, reporting and verification (MRV). It reveals inconsistencies between economic development and forest protection, as well as practical difficulties stressing the need for land reform and land planning.
\end{abstract}

\section{Introduction}

L'acronyme REDD+ s'est rapidement diffusé ces dernières années dans les communautés scientifiques qui $\mathrm{s}^{\prime}$ intéressent aux forêts tropicales ${ }^{1}$. Il désigne la réduction des émissions de gaz à effet de serre (EGES) résultant de la déforestation et de la dégradation des forêts dans les pays en développement, le " plus » signifiant la prise en compte des activités de conservation et de gestion durable des forêts, ainsi que l'accroissement des stocks de

\footnotetext{
Auteur correspondant : M. Tsayem Demaze, moise.tsayem_demaze@univ-lemans.fr

1 Cet article a été préparé dans le cadre du projet Demiccac (Déforestation évitée et mitigation du changement climatique en Afrique centrale), cofinancé par l'Institut de recherche pour le développement (IRD), l'université du Maine et l'université de Yaoundé I.
} 


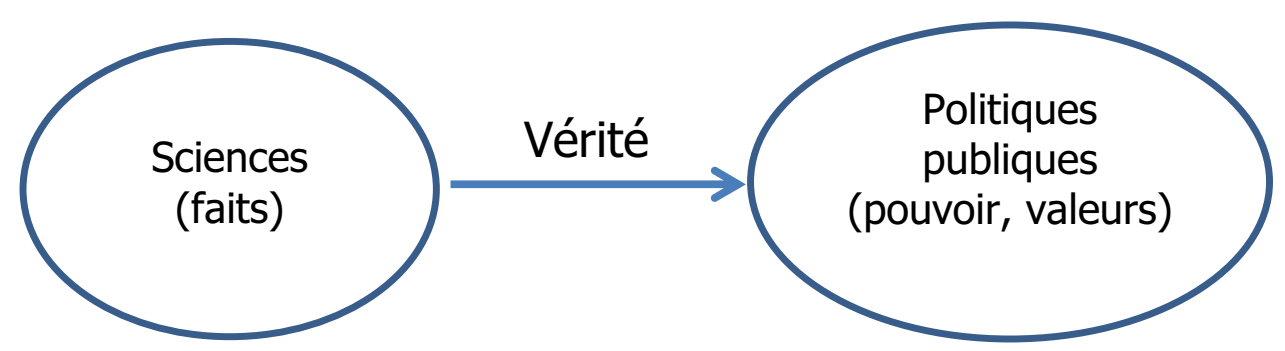

Fig. 1. Modèle linéaire conventionnel de la relation entre science et politique publique (d'après Kamelarczyk, et al., 2014).

carbone forestier. La REDD+ est élaborée dans les négociations internationales appelées conférences des parties (COP). Elles rassemblent chaque année les États qui ont ratifié la convention-cadre des Nations unies sur le changement climatique. Contrairement aux forêts tempérées dont les surfaces ont augmenté entre 1990 et 2005, les forêts tropicales continuent d'être l'objet d'une déforestation et d'une dégradation qui, par les EGES, sont pointées du doigt comme étant contributrices du changement climatique. D'après van der Werf et al. (2009), la déforestation est à $1^{\prime}$ origine de $12 \%$ à $15 \%$ des EGES dans le monde.

Le principe de la REDD+ est d'inciter les pays en développement soit à réduire la déforestation ou la dégradation des forêts, soit à gérer durablement les forêts ou à augmenter les stocks de carbone forestier, étant entendu que, s'ils y parviennent, ils pourront être récompensés financièrement (Karsenty et Pirard, 2007).

La conception de la REDD+ au fil des COP s'inscrit dans les négociations pour la période post-Kyoto 1 qui a commencé en 2013, sans rupture fondamentale avec la période Kyoto 1 (2008-2012). Dans cette perspective, la REDD+, d'un point de vue géopolitique, peut être considérée comme un mécanisme visant à impliquer davantage les pays en développement dans la lutte contre le changement climatique, bien que les modalités d'engagement restent incertaines, tout comme celles des pays développés, vis-à-vis de leurs EGES. Une clarification et une structuration beaucoup plus abouties sont espérées à l'issue de la COP 21 à Paris en décembre 2015.

De quels travaux scientifiques la construction et la mise en œuvre de la REDD+ se nourrissent-elles ? Comment la REDD+ est-elle appropriée et mise en œuvre par les pays en développement?

Cet article retrace d'abord la genèse de la REDD+ pour montrer les éventuels liens entre les travaux des scientifiques et les décisions des pouvoirs publics entendus au sens de représentants des États participant aux COP. Pour cela, le suivi des COP est couplé à l'analyse de la bibliographie relative à la REDD+. Cette partie de l'article s'inspire des démarches analytiques utilisées pour étudier les interactions entre sciences et politiques publiques (Kamelarczyk et Smith-Hall, 2014). Elle s'inspire en outre de l'analyse institutionnelle discursive (AID) utilisée notamment par den Besten et al. (2014) pour identifier les coalitions discursives représentées par diverses catégories d'acteurs ayant participé à l'institutionnalisation de la REDD+.

L'article aborde ensuite la mise en œuvre de la REDD+ dans des États de l'Afrique centrale, en s'appuyant sur la lecture comparative de leurs plans de préparation (readiness preparation proposals), ainsi que sur notre expérience dans l'élaboration du plan de préparation du Cameroun. Cette deuxième partie prolonge et complète la première en illustrant les difficultés relatives à la mise en œuvre, à l'échelle sous-régionale et nationale, de la REDD+ élaborée au niveau mondial.

\section{La conception de la REDD+ : une coproduction par les pouvoirs publics et les scientifiques ?}

\section{Cadre analytique}

On considère généralement que, pour traiter un problème, les politiques sont élaborées à partir de connaissances scientifiques. Selon cette conception des relations entre sciences et pouvoirs publics, la production du savoir scientifique interviendrait en amont du processus politique, par un simple transfert de connaissances, sans que les acteurs scientifiques ne soient directement impliqués dans les décisions (Fig. 1).

Ce modèle linéaire est-il pertinent pour décrire la conception de la REDD+? D'après Kamelarczyk et Smith-Hall (2014), les recherches en political ecology et en sciences and technologies studies ont montré une forte imbrication entre production du savoir scientifique et élaboration des politiques publiques dans le domaine de l'environnement. Ces recherches mettent en évidence l'importance des contextes politiques et sociaux, qui font que les politiques environnementales sont largement le résultat d'une coproduction à travers l'acquisition, la diffusion et l'application des connaissances. D'après notre 


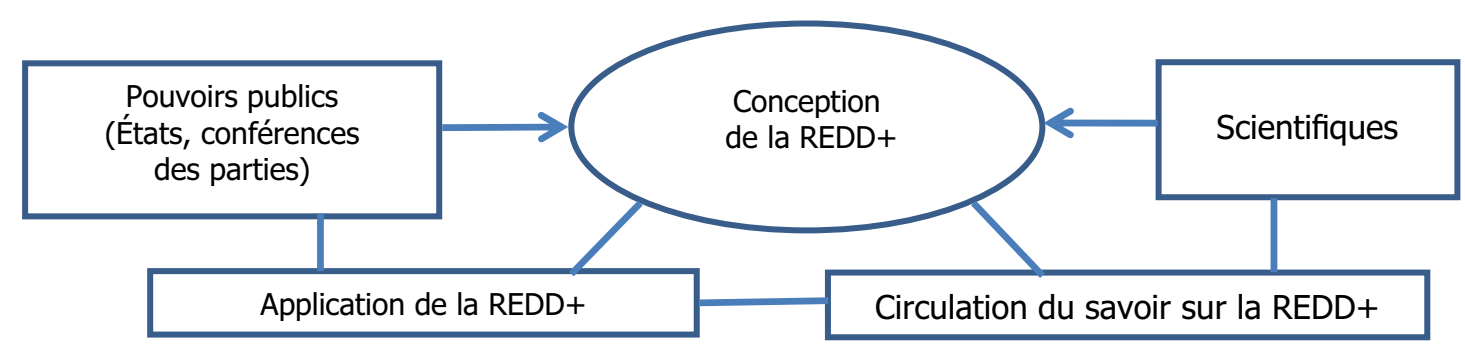

Fig. 2. REDD+ vue comme un savoir produit par des pouvoirs publics et des scientifiques (d'après Kamelarczyk, et al., 2014).

expérience du suivi des COP, la conception de la REDD+ ne correspond pas au modèle linéaire. Elle se nourrit d'échanges entre pouvoirs publics et scientifiques (Fig. 2). Les pouvoirs publics prennent généralement les devants et consolident progressivement la REDD+ avec des connaissances scientifiques (premier niveau du schéma, avec les flèches indiquant que REDD+ est le résultat d'une construction impliquant les pouvoirs publics et les scientifiques). La circulation du savoir, ainsi que sa mise en œuvre (deuxième niveau du schéma) nourrissent mutuellement les pouvoirs publics et les scientifiques, contribuant sans cesse à la conception du mécanisme.

Comment ce processus s'institutionnalise-t-il concrètement? Les études sur l'élaboration des politiques environnementales ont montré comment l'énoncé de problèmes environnementaux résulte de processus discursifs (den Besten et al., 2014). L'analyse institutionnelle discursive utilisée par ces auteurs prend en compte le contexte dans lequel les discours émergent pour devenir des politiques ou des pratiques institutionnalisées, à la suite d'alliances et de confrontations entre des groupes d'acteurs en compétition pour faire (pré)valoir leurs points de vue. En appliquant l'AID à l'étude de la conception de la REDD+, den Besten et al. (2014) montrent comment des coalitions discursives associant diverses catégories d'acteurs ont été constituées entre 2004 et 2011, donnant lieu à une spirale, c'est-à-dire un lien qui entremêle successivement, d'une part les idées émises par les acteurs, d'autre part les décisions politiques. Notre article reprend cette approche en ciblant spécifiquement les acteurs scientifiques et en élargissant la période étudiée. Nous mettons ainsi en parallèle, sur la période 1997-2014, l'évolution des problématiques et des concepts dominant la production scientifique sur la REDD+, et l'évolution de sa conception politique. La production scientifique examinée a été collectée sur les plateformes CAIRN et Science Direct en octobre 2014. En utilisant le mot-clé REDD, 94 et 260 références ont été obtenues respectivement. La lecture des titres et des résumés de ces articles a permis de sélectionner ceux (une soixantaine) qui abordent les aspects conceptuels de la REDD+ et sa mise en œuvre en Afrique centrale.
Cette sélection, sans être exhaustive, nous paraît représentative des recherches sur ce mécanisme, la principale limite étant qu'il ne s'agit que d'articles publiés soit en anglais, soit en français.

\section{Les trois phases de construction de la REDD+ et les préoccupations scientifiques associées}

Notre découpage en trois phases découle du suivi des COP. À chaque phase, nous associons des concepts et des thématiques reflétant les préoccupations scientifiques (Fig. 3). Le panorama élaboré permet d'interroger les liens entre sciences et politiques, ainsi que les coalitions discursives éventuelles. Notre découpage est différent de celui de den Besten et al. (2014) qui ne traite pas la phase opérationnelle et s'arrête en 2008. Le nôtre part de l'adoption du protocole de Kyoto (1997), et prend en compte la totalité des COP. Il considère 2009-2010 comme une période-clé : adoption du sigle REDD+ et démarrage de la phase opérationnelle.

\section{La phase 1, de 1997 à 2004 : la période pré-RED}

À la suite de l'adoption du protocole de Kyoto en 1997 (COP 3), des négociations houleuses eurent lieu pour intégrer les forêts tropicales dans les mécanismes de flexibilité du protocole (Jung, 2005). Ces négociations opposèrent notamment les États-Unis - qui plaidaient pour - aux pays de l'Union européenne - qui étaient contre, en raison principalement des difficultés techniques et des craintes relatives à l'efficacité du dispositif et à la complexité de la question de la déforestation dans les pays en développement. Seuls les projets de boisement/reboisement ont finalement été admis au MDP (mécanisme pour un développement propre), un des mécanismes de flexibilité du protocole. Mais le très faible nombre de ces projets montre que les enjeux forestiers dans les pays en développement ne sont pas véritablement traités dans le MDP (Tsayem, 2012). C'est pour traiter ces enjeux que le concept de " réduction compensée » des EGES émergea en 2003, lors de la COP 9 à Milan (Moutinho et Schwartzman, 2005). Il fut élaboré notamment par l'Institut de recherche environnementale de l'Amazonie (Ipam). Il suggérait d'encourager la lutte 


\begin{tabular}{|c|c|c|c|}
\hline & \multicolumn{3}{|c|}{$\begin{array}{lll}\text { Phase 1 (1997-2004) } & \text { Phase 2 (2005-2009) } & \begin{array}{l}\text { Phase 3 (depuis 2010) } \\
\text { (mode opératoire REDD }+ \text { ) }\end{array} \\
\text { Période pré-RED } & \text { (de la RED à la REDD+) } & \text { (mode }\end{array}$} \\
\hline $\begin{array}{l}\text { Pouvoirs publics } \\
\text { (décisions, } \\
\text { politiques } \\
\text { publiques) }\end{array}$ & $\begin{array}{l}\text { De la COP3 à la } \\
\text { COP10, } \\
\text { protocole de Kyoto, } \\
\text { mécanisme de } \\
\text { flexibilité, accords de } \\
\text { Marrakech (2001) sur } \\
\text { le MDP intégrantle } \\
\text { boisement/ } \\
\text { reboisement, etc. }\end{array}$ & $\begin{array}{l}\text { De la COP11 à la COP15, } \\
\text { RED (2005), REDD (2007), } \\
\text { REDD+ (2009), création } \\
\text { et/ou développement des } \\
\text { marchés du carbone (MDP, } \\
\text { MOC, SC EQE,...), } \\
\text { programmes REDD+ de la } \\
\text { Banque Mondiale et de } \\
\text { PONU, Fonds multilatéraux } \\
\text { et bilatéraux, etc. }\end{array}$ & $\begin{array}{l}\text { Depuis la COP } 16 \text {, } \\
\text { élaboration, validation et adoption des } \\
\text { programmes de préparation, } \\
\text { élaboration des stratégies nationales, } \\
\text { coopération multilatérale et bilatérale, } \\
\text { déploiement des fonds, garde-fous } \\
\text { environnementaux et sociaux, } \\
\text { renforcement des capacités des pays } \\
\text { en développement, projets pilotes, } \\
\text { cadre juridique de mise en ouvre, etc. }\end{array}$ \\
\hline $\begin{array}{l}\text { Scientifiques } \\
\text { (problématiques } \\
\text { et concepts) }\end{array}$ & $\begin{array}{l}\text { Puits de carbone, } \\
\text { afforestation/ } \\
\text { reforestation, } \\
\text { réduction compensée } \\
\text { des émissions, etc. }\end{array}$ & $\begin{array}{l}\text { Options politiques, } \\
\text { architectures institutionnelles, } \\
\text { positionnements théoriques } \\
\text { et postures critiques, } \\
\text { additionnalité de la réduction } \\
\text { des émissions, } \\
\text { coûts/bénéficies financiers et } \\
\text { environnementaux, } \\
\text { faisabilité de la mesure des } \\
\text { stocks et des flux de carbone } \\
\text { associés aux forêts et à leur } \\
\text { déforestation/dégradation, } \\
\text { prototypes de projets pilotes } \\
\text { (expérimentation), etc. }\end{array}$ & $\begin{array}{l}\text { Services environnementaux et } \\
\text { paiements pour, coûts d'opportunité, } \\
\text { économie politique, capacités } \\
\text { institutionnelles, géopolitique, sciences } \\
\text { politiques, gouvernance, sciences } \\
\text { studies, political ecology, techniques et } \\
\text { méthodes de mesure des stocks et des } \\
\text { flux de carbone, scénarios de référence } \\
\text { et lignes de base, évaluation des projets } \\
\text { pilotes et des programmes de } \\
\text { préparation, participation, droits et } \\
\text { revenus du carbone, droit des } \\
\text { populations autochtones, droits } \\
\text { fonciers, financements, etc. }\end{array}$ \\
\hline
\end{tabular}

MDP: Mécanisme pour un développement propre; MOC: Mise en œuvre conjointe; SC EQE: Système communautaire d'échange des quotas d'émissions (marché carbone de l'Union européenne).

Fig. 3. Pouvoirs publics et sciences dans les principales phases de conception de la REDD+.

contre la déforestation en récompensant financièrement les pays en développement qui parviendraient de manière prouvée à réduire volontairement les EGES résultant de cette déforestation.

L'Ipam est une structure de recherche qui fonctionne comme un think tank spécialisé sur l'Amazonie brésilienne. Il participe aux COP et intervient dans l'expertise REDD+ au Brésil. Ses activités de recherche nourrissent et orientent les débats et les négociations, notamment pour l'élaboration de la stratégie REDD+ du Brésil. Entre 2010 et 2012, l'Ipam a siégé au conseil de direction du programme REDD de l'Onu, en tant que représentant des organisations de la société civile de la zone Amérique latine et Caraibes. Son lobbying ${ }^{2}$ est une illustration des liens entre sciences et décideurs politiques participant à l'élaboration de la REDD+. D'autres travaux scientifiques ont abordé, au cours de la phase 1, la question de la mesure des flux annuels de carbone, en lien avec l'afforestation, agissant comme puits de carbone, et avec la déforestation, agissant comme source d'émissions de carbone (Houghton, 1999).

2 Voir http://www.ipam.org.br/.

\section{La phase 2, de 2005 à 2009 : de la RED à la REDD+}

Le concept de réduction compensée a été repris par le Costa Rica et la Papouasie-Nouvelle-Guinée, qui ont présenté lors de la COP 11 à Montréal en 2005, une proposition suggérant des incitations pour encourager la réduction des émissions issues de la déforestation (RED) dans les pays en développement. Cette proposition était soutenue par une coalition de pays appartenant à la zone forestière humide ${ }^{3}$. La première formulation de la RED fut présentée dans un contexte spécifique : le protocole de Kyoto était entré en vigueur un an avant, et les pays l'ayant ratifié se réunissaient pour la première fois, dans le cadre du Meeting of Parties (MOP), conjointement avec la COP. En outre, des négociations s'ouvraient pour une deuxième période d'application du protocole. Le Brésil a ensuite présenté des propositions de RED, notamment lors de la COP 12 en 2006 à Nairobi. Elles suggéraient des récompenses financières, sur fonds publics, pour encourager l'application de mesures aboutissant à la réduction de la déforestation dans les pays en développement, les fonds publics devant être alimentés

\footnotetext{
3 Voir http://rainforestcoalition.org/.
} 
par les pays développés. Les négociations lors de la COP 13, à Bali en 2007, ont consacré une grande place à la RED, sans doute parce que le quatrième rapport du GIEC, publié peu avant cette COP, avait souligné le fait que la déforestation représentait entre $11 \%$ et $28 \%$ des émissions de carbone dans le monde. Le rapport Stern, publié en 2006, évoquait un pourcentage proche. Ces éléments de contexte illustrent l'influence que des scientifiques (GIEC, Stern) ont pu exercer directement ou indirectement sur les pouvoirs publics participant à l'élaboration de la REDD+ dans les COP.

À la demande des pays du bassin du Congo, un deuxième « $\mathrm{D}$ », signifiant « dégradation des forêts », fut adopté lors de la COP 13, afin que la déforestation de faible ampleur consécutive à la coupe sélective du bois en Afrique centrale soit prise en compte. On est alors passé de la RED à la REDD. D'autres éléments (conservation, gestion durable, stockage du carbone) ont ensuite été incorporés, essentiellement à la demande de la Chine et de l'Inde, qui souhaitaient que la création d'aires protégées et la sylviculture soit prise en considération. La COP 13 appelait aussi au démarrage de projets pilotes ou expérimentaux, et suggérait que les pays développés soutiennent financièrement ces initiatives REDD, pour que les résultats soient intégrés dans l'élaboration du traité international que les États devaient adopter à Copenhague en 2009 (COP 15). Bien que la COP 15 n'ait pas débouché sur le traité qui était espéré, elle a consacré l'évolution du périmètre de la REDD, avec un + relatif aux éléments adoptés lors des COP 13 et 14 . Depuis lors, c'est le sigle REDD+ qui prévaut. Des propositions ont été faites pour ajouter un deuxième plus (REDD++) afin que soient prises en compte des activités comme l'agroforesterie ou la plantation/gestion d'arbres dans les espaces non forestiers (savanes, montagnes). Mais elles n'ont pas eu de succès, tout comme des propositions d'élargissement émanant de scientifiques (Robiglio et al., 2010) développant le concept de REALU (Reducing Emissions from All Land Uses).

La conception de la REDD+ a entraîné un élargissement des marchés carbone, en particulier les marchés volontaires : Verified Carbon Standard (VCS), Plan Vivo (PV), Community, Climate and Biodiversity Alliance (CCBA), etc. Ils intègrent les crédits carbone issus des projets REDD+. Des programmes de soutien financier aux pays en développement ont été mis en œuvre. La Banque mondiale a créé plusieurs programmes REDD+, dont le plus important, en matière de participation des pays en développement, est le Fonds de partenariat pour le carbone forestier (FPCF), opérationnel depuis 2008. La même année, les Nations unies ont créé le programme Onu-REDD, qui soutient aussi financièrement les pays en développement qui se préparent à la REDD+. Mêlant coopérations bilatérale et multilatérale, associant des pays développés et des pays en développement, ainsi que des experts et consultants, des organisations non gouvernementales et des organisations de la société civile, ces programmes institutionnalisent la REDD+ avec des approches divergentes, mais complémentaires (Tsayem, 2012).

Les travaux scientifiques qui ont alimenté les débats lors du passage de la RED à la REDD+ ont entériné ce passage et ont produit des analyses abordant les options politiques et les architectures institutionnelles pour le fonctionnement de la REDD+ (Pedroni et al., 2009; Karsenty, 2008), les rémunérations financières, les dimensions économiques relatives aux coûts d'opportunité, aux effets d'aubaine, aux bénéfices financiers et environnementaux (Bellassen et Gitz, 2008 ; Karsenty, 2008 ; Karsenty et Pirard, 2007). D'autres travaux, à caractère méthodologique (traitement $\mathrm{d}^{\prime}$ images satellites), ont porté sur la fiabilité de la mesure de la déforestation en évaluant les stocks et les flux de carbone associés (DeFries et al., 2007). Les problématiques concernant le caractère additionnel ou non de la REDD+ ont aussi été abordées (Moutinho et Schwartzman, 2005), tout comme les premiers projets pilotes (Fergusson, 2009). Des postures critiques et des positionnements théoriques montrant les limites de la marchandisation du carbone ont été exprimés dans certains travaux scientifiques (Karsenty et Pirard, 2007). Ces travaux remettent en cause la « réduction compensée » des EGES qui est à l'origine de la REDD+ envisagée en lien avec les marchés carbone et les financements internationaux (Moutinho et Schwartzman, 2005).

\section{La phase 3, depuis 2010 : mode opératoire de la REDD+}

Entamée en 2010 avec les accords de Cancún (COP 16), la phase 3 porte essentiellement sur le mode opératoire de la REDD+. Les COP qui se succèdent consacrent une grande part des débats aux modalités pratiques et à la mobilisation des fonds. Les coopérations multilatérales et bilatérales se déploient pour aider les pays en développement à élaborer des programmes de préparation et des stratégies nationales, en suivant le format des guidelines et garde-fous environnementaux et sociaux prônés notamment par le FPCF et par OnuREDD. Ces programmes enrôlent des pays en développement candidats à la préparation à la mise en œuvre de la REDD+. Alors que des pays développés se consacrent à la mobilisation des fonds, des pays en développement se chargent d'entreprendre des réformes pour incorporer le dispositif REDD+ et ce qu'il regorge en matière d'exigences de plus en plus croissantes, notamment sur les plans fonciers, juridiques, institutionnels et même socio-économiques. Les fonds multilatéraux et bilatéraux, par exemple ceux mis en place par la Norvège, renforcent l'institutionnalisation de la REDD+ et contribuent à la rendre opérationnelle dans 
une perspective de coopération Nord-Sud visant à renforcer les capacités des pays en développement (Tsayem, 2012). Divers acteurs, essentiellement en provenance des pays développés (ONG de conservation de la nature, bureaux d'études, consultants internationaux), participent à cette coopération, pour développer des projets pilotes et fournir aux pays en développement l'expertise multiple et complexe que la mise en œuvre de la REDD+ requiert. En se projetant progressivement des arènes de négociation (COP) au terrain (pays en développement), la REDD+ s'apparente de plus en plus à l'aide publique au développement (assistance technique et financière des pays développés).

La production scientifique porte sur plusieurs approches, avec des travaux abordant les dimensions économiques ou de politiques économiques: coûts d'opportunité, effet d'aubaine, coûts de transaction, liens avec les paiements pour services environnementaux, apports aux marchés carbone (Mahanty et al., 2013 ; Karsenty, 2012). Les dimensions politiques, géopolitiques et institutionnelles donnent aussi lieu à de nombreuses publications (Korhonen-Kurki et al., 2014; Aquino et Guay, 2013 ; Somorin et al., 2013 ; Angelsen et al., 2010 ; Karsenty et Ongolo, 2012 ), tout comme les dimensions techniques focalisées sur la télédétection et les mesures des stocks et flux de carbone, le monitoring et les scénarios de référence pour l'évolution projetée de la déforestation (De Sy et al., 2012). Les recherches à caractères économétrique (Bellassen et Gitz, 2008) et méthodologique (DeFries et al., 2007), entérinant le principe de la REDD+, l'envisagent avec pragmatisme et accompagnent la construction politique du mécanisme. Des organismes internationaux (Banque mondiale, $\mathrm{Onu}$ ), ainsi que des ONG internationales de conservation de la nature, en déployant leurs programmes et leurs projets REDD+ dans les pays en développement, intègrent la dimension marchande. Des organismes de certification (VCS, PV, CCBA), jouant un rôle d'intermédiaires entre les porteurs de projets REDD+ et de potentiels acquéreurs de crédits carbone, mettent en œuvre des méthodes de comptabilité carbone pour permettre les transactions dans les marchés volontaires.

De nouvelles approches scientifiques se développent. C'est le cas de l'approche science studies, analysant la manière donc les sciences et techniques sont utilisées pour construire et légitimer la REDD+ (Kamelarczyk et Smith-Hall, 2014 ; Holmgren, 2013 ; den Besten et al., 2014 ; Bidaud, 2012). C'est aussi le cas de l'approche consistant à évaluer les projets pilotes et les programmes de préparation des pays en développement (Awono et al., 2014 ; Akong Minang et al., 2014 ; Aquino et Guay, 2013 ; Murdiyarso et al., 2012). Cette catégorie de travaux insiste notamment sur les difficultés pratiques de la REDD+, la problématique de la participation des acteurs, en particulier les populations locales/autochtones, les rémunérations liées aux marchés carbone, ou les droits fonciers. La REDD+ commence à donner lieu à des analyses sociologiques et ethnographiques. Elles s'intéressent au profil des experts. Comme les «courtiers» du développement dans les années postcoloniales, ils préparent les pays en développement. Ehrenstein (2013) les désigne, en République démocratique du Congo (RDC), par l'expression "professionnels de la préparation », en décrivant la vacuité de «ce que signifie préparer». L'émergence de ce type de recherche montre que, même si le savoir scientifique a pu inspirer la construction politique de la REDD+, il est maintenant porté sur une analyse critique en montrant l'incongruité et les difficultés opérationnelles de la REDD+ (Akong Minang et al., 2014). Ce sont surtout les sciences humaines et sociales, encore minoritaires, qui sont dans cette posture.

Comment la REDD+ est-elle transcrite et appliquée sur le terrain lorsqu' on passe de sa conception dans les arènes internationales à son appropriation par les pays en développement? Nous procédons ci-après à une description de la préparation à la REDD+ en Afrique centrale. L'objectif n'est pas de prolonger l'analyse institutionnelle discursive et l'étude des interactions entre sciences et politiques, mais de rendre compte des difficultés inhérentes à l'opérationnalisation de la REDD+.

\section{La préparation à la REDD+ en Afrique centrale}

\section{État d'avancement et défis}

Les pays du bassin du Congo engagés dans la préparation à la REDD+ suivent les directives-cadres du FPCF. Bien que l'engouement des pays soit comparable, l'engagement effectif est à des stades différents (Tab. 1). La note d'intention (R-PIN) du Gabon a été approuvée par le FPCF en 2008, ce qui lui a permis d'obtenir des fonds (200 000 \$ US) pour son plan de préparation (R-PP). Il n'est pas encore approuvé, alors que ceux des autres pays sont au stade de la mise en œuvre ${ }^{4}$. La RDC est le pays le plus avancé dans la préparation (Aquino et Guay, 2013), même si celle-ci peine à se traduire en réalité concrète (Ehrenstein, 2013). Son R-PP a été approuvé en 2010, par le FCPF et par Onu-REDD. Sa stratégie nationale REDD+ a été validée en 2014. L'élaboration de la stratégie nationale, le développement d'un niveau de référence des émissions, et la mise en place d'un système national de mesures, notifications et vérifications (MNV), constituent l'essentiel des activités de préparation.

\footnotetext{
4 Les R-PP sont disponibles sur le site https:/ / www.forestcarbonpartnership.org/.
} 
Tableau 1. Panorama de la préparation à la REDD+ en Afrique centrale.

\begin{tabular}{|c|c|c|c|c|c|}
\hline & RCA & Cameroun & Congo & RDC & Gabon \\
\hline $\begin{array}{l}\text { Approbation } \\
\text { du R-PP }\end{array}$ & mai 2013 & janvier 2013 & septembre 2011 & juillet 2010 & mai 2008 (R-PIN) \\
\hline $\begin{array}{l}\text { Leadership } \\
\text { politico- } \\
\text { administratif }\end{array}$ & $\begin{array}{l}\text { ministère de } \\
l^{\prime} \text { Environnement et } \\
\text { de l’Écologie }\end{array}$ & $\begin{array}{l}\text { ministère } \\
\text { de l'Environnement, } \\
\text { de la Protection } \\
\text { de la nature } \\
\text { et du Développement } \\
\text { durable }\end{array}$ & $\begin{array}{l}\text { ministère } \\
\text { du Développement } \\
\text { durable, } \\
\text { de l'Économie } \\
\text { forestière } \\
\text { et de l'Environnement }\end{array}$ & $\begin{array}{l}\text { ministre } \\
\text { de l'Environnement, } \\
\text { Conservation } \\
\text { de la nature } \\
\text { et du Tourisme }\end{array}$ & $\begin{array}{l}\text { ministère } \\
\text { de l'Environnement, } \\
\text { du Développement } \\
\text { durable } \\
\text { et de la Protection } \\
\text { de la nature }\end{array}$ \\
\hline $\begin{array}{l}\text { Structures } \\
\text { chargées des } \\
\text { aspects } \\
\text { opérationnels }\end{array}$ & $\begin{array}{l}\text { comité national, } \\
\text { comités } \\
\text { interpréfectoraux, } \\
\text { coordination } \\
\text { technique }\end{array}$ & $\begin{array}{l}\text { comité de pilotage, } \\
\text { secrétariat } \\
\text { technique, } \\
\text { coordinations } \\
\text { régionales, comités } \\
\text { techniques } \\
\text { départementaux }\end{array}$ & $\begin{array}{l}\text { comité national, } \\
\text { coordination } \\
\text { nationale, comités } \\
\text { départementaux }\end{array}$ & \begin{tabular}{l|} 
comité national, \\
comité \\
interministériel, \\
coordination nationale
\end{tabular} & \\
\hline $\begin{array}{l}\text { Groupes } \\
\text { de parties } \\
\text { prenantes-clés }\end{array}$ & \multicolumn{4}{|c|}{$\begin{array}{l}\text { société civile, ONG nationales et internationales, secteur privé, administrations centrales } \\
\text { et déconcentrées, collectivités territoriales décentralisées, médias, élus, bailleurs de fonds, } \\
\text { partenaires au développement, partenaires techniques et financiers }\end{array}$} & \\
\hline $\begin{array}{l}\text { Modalités } \\
\text { de travail }\end{array}$ & \begin{tabular}{|l} 
entretiens \\
individuels, \\
ateliers nationaux, \\
provinciaux, \\
ateliers pour les \\
populations locales
\end{tabular} & $\begin{array}{l}\text { consultations, ateliers } \\
\text { aux niveaux national, } \\
\text { régional et local, } \\
\text { et dans chaque zone } \\
\text { agroécologique }\end{array}$ & $\begin{array}{l}\text { campagne nationale, } \\
\text { ateliers nationaux, } \\
\text { départementaux, } \\
\text { discussions, groupes } \\
\text { d'experts }\end{array}$ & $\begin{array}{l}\text { ateliers nationaux } \\
\text { ou provinciaux, } \\
\text { ateliers spécifiques }\end{array}$ & \\
\hline $\begin{array}{l}\text { Options } \\
\text { stratégiques } \\
\text { et défis } \\
\text { majeurs }\end{array}$ & \multicolumn{4}{|c|}{$\begin{array}{l}\text { - options sectorielles (amélioration des performances de productivité), } \\
\text { - options transversales (aménagement du territoire et zonages associés), } \\
\text { - s'attaquer aux « facteurs directs » de la déforestation et de la dégradation des forêts et aux } \\
\text { « causes sous-jacentes », } \\
\text { - mettre en adéquation les lois foncières et forestières et les systèmes de partage des bénéfices, } \\
\text { - réaliser l'évaluation environnementale et sociale stratégique (EESS), } \\
\text { - développement d'un niveau de référence et conception d'un système de suivi (MNV) } \\
\text { combiné aux scénarios prospectifs, } \\
\text { - participation effective des organisations de la société civile et des représentants } \\
\text { des populations des zones forestières, } \\
\text { - réformes institutionnelles et renforcement des capacités, } \\
\text { - intégrer l'agenda REDD+ et l'agenda d'émergence économique }\end{array}$} & \\
\hline $\begin{array}{l}\text { Calendrier } \\
\text { et budget }\end{array}$ & \multicolumn{4}{|c|}{$\begin{array}{l}\text { Programmation sur trois ans avec des besoins de base de } 20 \text { millions de } \$ \text { US en moyenne } \\
\text { par pays }\end{array}$} & \\
\hline
\end{tabular}

De manière systématique, le premier écueil dans l'élaboration du R-PP, première phase de la préparation à la REDD+, est la recherche d'un arrangement institutionnel pour intégrer toutes les parties prenantes, et susciter une forte collaboration intersectorielle, afin de s'assurer les soutiens politiques, techniques et financiers nécessaires. La tendance a été la mise en place de comités interministériels de pilotage regroupant toutes les institutions concernées. Une dualité a alors été créée entre une instance administrative délibérante et une coordination technique. Au Cameroun, un arbitrage a été effectué par le Premier ministre, qui a confié le leadership de la préparation au ministère en charge de l'environnement (dès le départ au premier plan de la participation du
Cameroun aux (OP) avec la collaboration du ministère en charge des forêts. Bien que la société civile soit conviée, le problème se trouve au niveau de sa représentativité : comment avoir des interlocuteurs crédibles? $\mathrm{Au}$ Cameroun, l'administration leader (ministère en charge de l'environnement) n'a pas hésité à impulser, de manière prudente, l'organisation de la société civile, en jouant comme un des facilitateurs de sa participation au processus REDD+. Par la suite, elle a fortement été impliquée dans le processus d'élaboration du R-PP. Ainsi, avec une société civile acquise à sa cause, le R-PP du Cameroun a été approuvé dès sa première soumission au FPCF, contrairement aux R-PP des autres pays (RDC, Congo, République centrafricaine). 
Tableau 2. Engouement et risques pour la REDD+ en Afrique centrale.

\begin{tabular}{|c|c|c|c|}
\hline Forces & Faiblesses & Opportunités & Menaces \\
\hline $\begin{array}{l}\text { Existence du potentiel forestier; } \\
\text { Volonté commune de valoriser } \\
\text { le patrimoine forestier ; } \\
\text { Existence d'institutions } \\
\text { sous-régionales (Commission } \\
\text { des forêts d'Afrique centrale); } \\
\text { Présence de partenaires } \\
\text { internationaux. }\end{array}$ & $\begin{array}{l}\text { Inventaires forestiers } \\
\text { parcellaires ou mal conduits ; } \\
\text { Zonage incomplet des } \\
\text { territoires nationaux; } \\
\text { Insuffisance de coordination } \\
\text { intersectorielle ; } \\
\text { Insuffisance des moyens } \\
\text { techniques et financiers. }\end{array}$ & $\begin{array}{l}\text { Intérêt porté au niveau } \\
\text { international sur la convention- } \\
\text { cadre des Nations unies sur le } \\
\text { changement climatique et sur la } \\
\text { REDD+ ; } \\
\text { Projet de zonage en débat dans } \\
\text { différents pays ; } \\
\text { Ambitions de développement. }\end{array}$ & $\begin{array}{l}\text { Incertitude du marché } \\
\text { du carbone ; } \\
\text { Précarité du foncier ; } \\
\text { Conflits potentiels liés } \\
\text { aux systèmes de partage } \\
\text { des revenus ; } \\
\text { Risques de fuite ; } \\
\text { Rapidité des évolutions } \\
\text { technologiques ; } \\
\text { Insécurité et contingences } \\
\text { politiques. }\end{array}$ \\
\hline
\end{tabular}

De nombreuses réunions ont eu lieu dans chaque pays pour définir les options stratégiques et esquisser les contours de la mise en œuvre de la REDD+. Plusieurs problèmes cruciaux sont apparus : l'inadéquation entre les lois foncières et forestières, l'inexistence $d$ 'un cadre pour les rémunérations et le partage des bénéfices, la faiblesse des capacités techniques, les contradictions entre l'agenda REDD+ et l'agenda de politique économique axée sur l'exploitation des ressources naturelles pour accélérer la croissance et parvenir à moyen terme au stade de pays émergent. Les législations forestières sont en cours de révision. La régulation du foncier oscille entre le modèle traditionnel/coutumier et le modèle de droit écrit, ce qui traduit sa complexité ainsi qu'une dualité aboutissant, en pratique, à une dérégulation. Des études sont prescrites pour mieux cerner le concept de forêt, pour clarifier les nouveaux droits et pour définir les modalités de partage des bénéfices de la REDD+, dans un contexte où scientifiques et acteurs politiques se rendent compte du caractère lacunaire des connaissances et tentent mutuellement d'orienter les recherches sur les aspects opérationnels et la gouvernance. Les recherches en sciences sociales montrent que la résolution des questions foncières est cruciale pour la participation des communautés locales et leur rémunération (Awono et al., 2014).

Volet transversal, 1'aménagement du territoire apparaît insuffisamment pris en compte dans les plans de préparation. Les R-PP évoquent pourtant l'importance des plans de zonage. La RDC distingue trois catégories de zonage : un zonage calqué sur le découpage administratif et dont dépend la mise en place des institutions provinciales, un zonage forestier au niveau national, un zonage lié à la mise en place des projets pilotes. Au Cameroun, le découpage en cinq zones agroécologiques est retenu. Des zones de mise en œuvre de la REDD+ (ZOMO-REDD+) et des zones optimales d'action (ZOAREDD+) ont été conçues pour la prise en compte des spécificités des différentes zones agroécologiques. Du fait de l'étendue des forêts du bassin du Congo, des pays de la région affichent leur volonté de valoriser le potentiel forestier en mettant en œuvre la REDD+. La préparation se déroule dans un contexte marqué par des faiblesses et menaces variées (Tab. 2), dont l'incertitude, voire l'effondrement actuel, des marchés carbone.

\section{Partenaires financiers et techniques internationaux}

Chaque pays a budgétisé sa préparation à hauteur de 20 millions \$ US en moyenne, attendus du FPCF et d'autres bailleurs multilatéraux et bilatéraux. Ce budget moyen ne tient pas compte de celui des projets pilotes mis en œuvre essentiellement avec l'apport financier des ONG internationales comme le WWF (Fonds mondial pour la nature). La Comifac (Commission des forêts d'Afrique centrale), avec son plan de convergence, appuie les États de la région, en particulier dans le cadre du projet MNV (mesures, notifications, vérifications de l'évolution du couvert forestier) soutenu par la FAO. D'autres initiatives ont émergé : la création de l'Observatoire des forêts d'Afrique centrale (Ofac) par des États membres du Partenariat pour les forêts du bassin du Congo (PFBC), le projet de bases de données numériques pour le suivi des forêts (The Access Initiative) du World Ressources Institute, la facilitation de l'élaboration des stratégies d'implication des acteurs et des groupes vulnérables (femmes, peuples autochtones) par 1'UICN (Union internationale pour la conservation de la nature), aussi investie dans la production de connaissances et de données utiles pour comprendre la REDD+ et construire les stratégies nationales. Ces différents partenaires, en connexion avec des scientifiques et les États concernés, participent à l'institutionnalisation discursive et pratique de la REDD+ en Afrique centrale.

\section{Particularités du Cameroun}

La démarche du Cameroun pour élaborer son R-PP a été spécifique, du fait du recours presque exclusif aux chercheurs/consultants nationaux et à d'autres acteurs locaux, notamment ceux de la société civile. 
Des enseignants-chercheurs universitaires (notamment des géographes et des botanistes/écologues) et des chercheurs des instituts nationaux de recherche ont été mobilisés par la coordination nationale REDD+ au ministère en charge de l'environnement. Cette situation tranche avec celle de la RDC, qui s'est appuyée sur des experts internationaux (Ehrenstein, 2013). Une autre spécificité du Cameroun est le fait qu'il considère la REDD+ comme une opportunité de développement, grâce à la valorisation du rôle climatique des forêts, cette valorisation étant envisagée en termes d'économie verte (acquisition des fonds de contrepartie résultant des efforts pour réduire les EGES). Il est prévu que les rémunérations résultant de la REDD+ soient utilisées pour répondre aux préoccupations des secteurs-clés de développement (modernisation de l'agriculture, intensification de l'élevage). Par ailleurs, dans le cadre de sa stratégie de croissance et d'émergence économique à l'horizon 2035, le Cameroun mise sur l'exploitation des forêts et des ressources naturelles dont beaucoup sont localisées en zone forestière, ce qui va inévitablement entraîner la déforestation. Des initiatives REDD+ à vocation opérationnelle sont cependant développées en fonction des spécificités des zones agroécologiques. Les projets pilotes REDD+ en cours d'implémentation ambitionnent d'offrir des avantages importants aux peuples autochtones et aux communautés dépendant des forêts : fourniture de moyens alternatifs de subsistance, partage équitable de revenus générés par les réductions d'émissions. Dans la pratique, l'inadéquation du cadre juridique, la carence des moyens financiers, la non-opérationnalisation de l'Observatoire national des changements climatiques, l'insuffisance de communication et de coordination interministérielle, ainsi que l'absence d'un plan actualisé d'aménagement du territoire, et l'histoire des politiques forestières, ont des conséquences néfastes dans la maîtrise de l'organisation de l'espace, rendant difficile l'opérationnalisation de la REDD+ (Akong Minang et al., 2014 ; Awono et al., 2014 ; Dkamela et al., 2014).

\section{Conclusion}

Le périmètre de la REDD+ s'est progressivement élargi, faisant d'elle un melting pot incorporant une multitude de dimensions (économiques, techniques, juridiques, sociales, etc.). Ce qu'elle recouvre apparaît mouvant, et elle tend à devenir l'instrument de conservation des forêts tropicales tout en se préoccupant du développement économique et social. En se complexifiant, la REDD+, paradoxalement, semble occulter la réflexion sur les causes et l'ampleur réelles de la déforestation et de la dégradation des forêts tropicales, ainsi que la réflexion sur la signification opérationnelle de la gestion dite durable de ces forêts. Ces réflexions devraient intégrer les aspects prospectifs, indispensables pour envisager l'avenir de ces forêts. Les liens entre les multiples facettes de la REDD+ ne sont pas formulés de manière explicite dans sa conception politique. Les travaux scientifiques ne semblent pas non plus s'intéresser à ces liens, tant les recherches multi ou interdisciplinaires sur la REDD+ sont rares (Visseren-Hamakers et al., 2012). Les relations entre évolution de la déforestation, flux de carbone, EGES et augmentation des températures, qui doivent être étudiées en associant des spécialistes de l'écologie, de la biologie des organismes végétaux, de la biochimie, sont encore très rares.

En Afrique centrale, la préparation à la REDD+ apparaît multiforme, à l'image du concept. L'examen du contenu des documents de préparation montre que le recours aux sources et références scientifiques est assez timide. Les articles scientifiques constituent moins de $10 \%$ des références citées. En revanche, les rapports institutionnels (FAO, Ofac, Global Forest Watch, etc.) y sont largement cités. La préparation révèle la complexité de la REDD+ et l'ampleur des réformes à réaliser. Elle souligne les contradictions et les défis à relever, entre les impératifs de développement et ceux de la protection de l'environnement, tout en remettant à jour les problèmes sociopolitiques (par exemple la question des populations des zones forestières et leurs droits) et les questions de gouvernance et de fragilité de l'État (Dkamela et al., 2014 ; Karsenty et Ongolo, 2012). Dans ce contexte, la REDD+ perd en lisibilité, et il devient difficile de concevoir et de maîtriser le savoir-faire nécessaire pour sa mise en œuvre. Une baisse de l'engouement est perceptible depuis 2014 auprès de certains acteurs impliqués en Afrique centrale, en particulier les acteurs politicoadministratifs au Cameroun. Certains évoquent une lassitude consécutive à la faiblesse des financements, et aux pesanteurs et mésententes entre les institutions ministérielles et entre les États et les partenaires internationaux.

Si cet article ne précise pas dans quelle mesure la production scientifique a influencé directement la conception de la REDD+ et vice versa, il montre que sciences et politiques interagissent de plusieurs manières. Des scientifiques de l'Ipam ont été à l'origine du concept de réduction compensée de la déforestation, qui sous-tend la REDD+. Leurs travaux ont servi à esquisser la REDD+, donnant lieu aux négociations lors des COP 11 et 12 (Moutinho et Schwartzman, 2005). Ils participent aux COP et au programme REDD+ de la Banque mondiale, ainsi qu'aux débats sur la stratégie REDD+ du Brésil. Les méthodes de mesure du carbone recommandées par les COP sont issues des travaux scientifiques, ainsi que les approches économétriques qui ont donné lieu aux marchés carbone (Bidaud, 2012). Des politiques, que ce soient les États (Norvège par exemple) ou plus globalement les COP et les programmes multilatéraux, 
interviennent en amont pour financer des expertises et des recherches permettant d'avancer dans la conception et la mise en œuvre de la REDD+. Une structure en charge des questions scientifiques et technologiques assiste et conseille les décideurs politiques dans les COP. Des scientifiques élaborent parfois des documents que des pays en développement présentent dans des COP. C'est notamment le cas pour Madagascar (Bidaud, 2012). Pour la préparation à la REDD+ au Cameroun, un des auteurs de cet article a été engagé en tant qu'expertconsultant national, selon l'expression des politiques, pour appuyer la structure ministérielle en charge du RPP. Contribuant au renforcement du contenu scientifique des documents de préparation, il a pu apprécier la tension entre les aspirations politiques et les besoins scientifiques. Quelques travaux scientifiques sont publiés par des acteurs politiques participant aux COP ou aux programmes REDD multilatéraux, notamment celui de la Banque mondiale (Aquino et Guay, 2013).

Ces liens multiformes suggèrent que l'évolution de la conception de la REDD+ correspond davantage au modèle de coproduction des politiques publiques environnementales qu'au modèle linéaire. Sciences et politiques semblent s'entremêler très souvent, avec des rôles pouvant être interchangeables (le scientifique jouant un rôle politique et le politique pouvant être scientifique). L'institutionnalisation de la REDD+ montre également une coalescence d'acteurs divers : États, organismes de coopération et d'aide au développement, organisations de la société civile, scientifiques, etc. Le corpus bibliographique mobilisé pour cet article montre une prédominance de trois catégories de disciplines : les sciences et techniques de mesure du carbone, les sciences économiques, et les sciences politiques et juridiques. Les liens avec les acteurs politiques permettent à ces disciplines de bénéficier d'importants financements pour des recherches qui aident à la construction et à la mise en œuvre de la REDD+, les autres disciplines, en particulier en sciences humaines et sociales, étant peu présentes. L'acuité des problématiques d'aménagement du territoire devrait amener la géographie à être sollicitée particulièrement dans la phase actuelle de mise en œuvre de la REDD+.

\section{Remerciements}

Nous remercions l'IRD, l'université du Maine et l'université de Yaoundé I qui financent les recherches qui ont permis de rédiger cet article. Nous remercions en outre les évaluateurs dont les suggestions ont permis d'améliorer considérablement la version initiale.

\section{Références}

Akong Minang, P., Van Noordwijk, M., Duguma, L.A., Alemagi, D., Hoan Do, T., Bernard, F., Agung, P., Robiglio, V., Catacutan, D., Suyanto Suyanto, Armas, A., Aguad, C.S., Feudjio, M., Galudra, G., Maryani, R., White, D., Widayati, A., Kahurani, E., Namirembe, S., Leimona, B., 2014. REDD+ Readiness progress across countries: Time for reconsideration, Climate Policy, doi: 10.1080/14693062.2014.905822.

Angelsen, A., Brockhaus, M., Kanninen, M., Sills, E., Sunderlin, W.D., Wertz-Kanounnikoff, S. (Eds), 2010. Réaliser la REDD+ : options stratégiques et politiques nationales, Cifor, Bogor, Indonésie.

Aquino, A., Guay, B., 2013. Implementing REDD+ in the Democratic Republic of Congo: An analysis of the emerging national REDD+ governance structure, Forest Policy and Economics, 36, 71-79.

Awono, A., Somorin, O.A., Eba'a Atyi, R., Levang, P., 2014. Tenure and participation in local REDD+ projects: Insights from southern Cameroon, Environmental Science and Policy, 35, 76-86.

Bellassen, V., Gitz, V., 2008. Reducing emissions from deforestation and degradation in Cameroon: Assessing costs and benefits, Ecological Economics, 68, doi :10.1016/ j.ecolecon.2008.03.015.

Bidaud, C., 2012. Le carbone qui cache la forêt : la construction scientifique et la mise en politique du service de stockage du carbone des forêts malgaches. Thèse de doctorat, Institut de hautes études internationales et du développement, Genève.

DeFries, R., Achard, F., Brown, S., Herold, M., Murdiyarso, D., Schlamadinger, B., de Souza Jr, C., 2007. Earth observations for estimating greenhouse gas emissions from deforestation in developing countries, Environmental Science and Policy, 10, 385-394.

De Sy, V., Herold, M., Achard, F., Asner, G.P., Held, A., Kellndorfer, J., Verbesselt, J., 2012. Synergies of multiple remote sensing data and sources for REDD+ monitoring, Current Opinion in Environmental Sustainability, 4, 6, 696706.

Den Besten, J.W., Arts, B., Verkooijen, P., 2014. The evolution of REDD+: An analysis of discursive-institutional dynamics, Environmental Science \& Policy, 35, 40-48.

Dkamela, G.P., Brockhaus, M., Kengoum Djieni, F., Schure, J., Assembe Mvondo, S., 2014. Lessons for REDD+ from Cameroon's past forestry law: A political economy analysis, Ecology and Society, 19, 3, 30. Online: http:// dx.doi.org/10.5751/ES-06839-190330.

Ehrenstein, V., 2013. Les professionnels de la préparation. Aider la République démocratique du Congo à réduire sa déforestation: programme REDD+, Sociologies Pratiques, 27, 91-103.

Ferguson, B., 2009. REDD comes into fashion in Madagascar, Madagascar Conservation and Development, 4, 2, 132-137.

Holmgren, S., 2013. REDD+ in the making: Orders of knowledge in the climate-deforestation nexus, Environmental Science E Policy, 33, 369-377. 
Houghton, R.A., 1999. The annual net flux of carbon to the atmosphere from changes in land use, 1850-1990, Chemical and Physical Meteorology, 51, 2, 298-313.

Jung, M., 2005. The role of forestry projects in the clean development mechanism, Environmental Science E Policy, 8, 87-104.

Kamelarczyk, K.B.F., Smith-Hall, C., 2014. REDD herring: Espistemic community control of the production, circulation and application of deforestation knowledge in Zambia, Forest Policy and Economics, 46, 19-29.

Karsenty, A., 2008. The architecture of proposed REDD schemes after Bali: Facing critical choices, International Forestry Review, 10, 3, 443-457.

Karsenty, A., 2012. Forêts : des instruments économiques décevants, Économie appliquée, Questions pour Rio+20, 65, 2.

Karsenty, A., Pirard, R., 2007. Changement climatique : faut-il récompenser la "déforestation évitée »?, Natures Sciences Sociétés, 15, 4, 357-369.

Karsenty, A., Ongolo, S., 2012. Can "fragile states" decide to reduce their deforestation? The inappropriate use of the theory of incentives with respect to the REDD mechanism, Forest Policy and Economics, 18, 31-48.

Korhonen-Kurki, K., Sehring, J., Brockhaus, M., Di Gregorio, M., 2014. Enabling factors for establishing REDD+ in a context of weak governance, Climate Policy, 14, 2, 167-186.

Mahanty, S., Suich, H., Tacconi, L., 2013. Access and benefits in payments for environmental services and implications for REDD+: Lessons from seven PES schemes, Land Use Policy, 31, 38-47.

Moutinho, P., Schwartzman, S. (Eds), 2005. Tropical Deforestation and Climate Change, Brasilia, Ipam.
Murdiyarso, D., Brockhaus, M., Sunderlin, W.D., Verchot, L., 2012. Some lessons learned from the first generation of REDD+ activities, Current Opinion in Environmental Sustainability, 4, 6, 678-685.

Pedroni, L., Dutschke, M., Streck, C., Porru, M.E., 2009. Creating incentives for avoiding further deforestation: The nested approach, Climate Policy, 9, 2, 207-220.

Robiglio, V., Ngendakumana, S., Gockowski J., Yemefac, M., Tchienkoua, M., Mbile, P., Tchawa, P., Tchoundjeu, Z., Bolognesi, M., 2010. Reducing emissions from all land uses in Cameroon. Final national report, ICRAF, Nairobi (online: http:/ / www.asb.cgiar.org/PDFwebdocs/ CAMEROON_REALU.pdf).

Somorin, A.O., Visseren-Hamakers, I.J., Arts, B., Sonwa, D.J., Tiani, A.-M., 2013. REDD+ policy strategy in Cameroon: Actors, institutions and governance, Environmental Science $\mathcal{E}$ Policy, 35, 87-97 (online: http://dx.doi.org/10.1016/ j.envsci.2013.02.004).

Tsayem Demaze, M., 2012. Du développement propre à la déforestation évitée: géoscopie des relations Nord-Sud pour atténuer le changement climatique. HDR, Université du Maine, Le Mans.

Van der Werf, G.R. ,Morton, D.C., DeFries, R.S., Olivier, J.G.J., Kasibhatla, P.S., Jackson, R.B., Collatz, G.J., Randerson, J.T., 2009. $\mathrm{CO}_{2}$ emissions from forest loss, Nature Geoscience, 2, 737-738.

Visseren-Hamakers, I.J., Gupta, A., Herold, M., Pena-Claros, M., Vijge, M.J., 2012. Will REDD+ work? The need for interdisciplinary research to adress key challenges, Current Opinion in Environmental Sustainability, 4, 590-596. 